\title{
MICRO STATISTICAL DESCRIPTORS FOR GLAUCOMA DIAGNOSIS USING NEURAL NETWORKS
}

\author{
Mukil Alagirisamy, \\ Department of Electrical and Electronics Engineering, \\ Lincoln University College, \\ Malaysia. \\ mukil.a@lincoln.edu.my
}

Submitted: Sep, 28, 2020 Revised: Dec, 28, 2020 Accepted: Jan, 12, 2021

\begin{abstract}
A fully automatic Computer Aided diagnosis (CAD) of glaucoma is developed that aims to reduce the false positive detection rate and increasing the sensitivity of classification. It consists of three main steps: Region of Interest (ROI) extraction (Optic Disc (OD) region), feature extraction (micro textures) and classification using Linear Vector Quantizer-Artificial Neural Network (LVQ-ANN). The search area for glaucoma is the OD region wherein the cupping occurs, so in the first step ROI is extracted from the whole image. Feature extraction and classification are the most challenging tasks as the performance of the system depend both of them. Laws defined five spatial filters to extract micro-statistical estimators such as Level, Edge, Spot, Wave, and Ripple. Fundus images in three databases; DRISHTI-GS1, ORIGA, and RIM-ONE are classified using LVQ-ANN classifier. Results indicate the strength of the LVQ-ANN classifier for glaucoma diagnosis with sensitivity of 95.71\% (DRISHTI-GS1), 83.33\% (ORIGA) and 94.87\% (RIM-ONE).
\end{abstract}

Keywords: Glaucoma diagnosis, fundus image, micro textures, computer aided diagnosis, LVQ-ANN.

\section{INTRODUCTION}

CAD refers to software which analyzes diseases using medical images or signals which helps radiologists and clinicians to decrease observational oversights and false negative rates in interpreting medical images/signals. Several CAD systems have been proposed to automatically detect glaucoma, most of which are based on the extracted features from different domains and classification approaches.

The Regions of Convolutional Neural Network (RCNN) with rule-based automated disc localization method is employed for the OD feature extraction of retinal fundus image. Then its classification of the healthy or glaucomatous image is performed using Deep Convolutional Neural Network (DCNN) [1]. Optical disc features for retinal glaucoma are generated. The selected dataset parameters are automatically obtained using the CNN from raw fundus images and given to the SVM classifier model for normal or abnormal image classification [2]. Risk factors complicates the glaucoma classification much more and [3] explains how each risk interacts with open-angle glaucoma disease and its characteristics such as prevalence, incidence, progress, and therapy employing the CNN scheme.

The ocular hypotensive medications for the retinal image analysis employ the automated glaucoma diagnosis technique by extracting the texture, color, contextual or structural dataset features. The features extracted from the patients are fed into the back propagation neural network classifier for the classification of the fundus images [4]. Glaucoma classification by utilizing the grid color moment 
generic dataset features represents the visual appearance and holistic. The local features are extracted to mitigate misalignment due to shape and sample size variations of retina images [5]. The CAD-based glaucoma image classification using the holistic and the local deep feature integration technique to enhance the classification system aids the application of CNN. The best performance is obtained using the large-scale screening programs using a transfer learning scheme [6].

The increase in intraocular eye pressure leads to a gradual and irreversible loss of vision. The textural property of the retinal image with and without the glaucoma images has been retrieved [7]. Haralick feature characteristics train the BPNN classifier model to differentiate normal from the retina damaged by glaucoma [8]. DTI analysis typically gives information on the orientation of white matter fibers measuring the degree of anisotropic diffusion in the tensor of fundus images [9]. Each DTI measure is produced from a given optical radioactivity ROI based and divided into three cases: histogram, co-occurrence matrices, and laws of features. Independent Component Analysis (ICA) follows the data matrix decomposition method and its reconstruction for the feature extraction [10]. Classification characteristics of the retinal fundus images are supported with the 10 -fold cross-validation using the K-Nearest Neighbor (KNN) based SVM classifier.

The Neural Network (NN) classifier technique is employed to extract fundus images' texture feature extraction [11]. The feature datasets of the retinal optic cup localization of the ROI-based testing model utilizes the NN classifier for providing excellent precision. Attention-based CNN subnet improves the reliability and accuracy of glaucoma classification [12] utilizes the large-scale attentionbased glaucoma database with localization area feature extraction of the fundus images.

The main contribution in this paper is to present a CAD system for the automatic detection of glaucoma using fundus images which can help a radiologist to improve his/her job and increase the radiologist's certainty to diagnose glaucoma. The rest of the paper is as follows: Section 2 proposes a glaucoma diagnosis system using micro statistical features and LVQ-ANN classifier. Section 3 analyzes the performances of the system by varying the size of the codebooks. Section 4 concludes this study.

\section{METHODS AND MATERIALS}

The classification or learning system trained to recognize samples from a dataset using some previously labeled or classified samples from the same dataset is performing classification or supervised learning. Supervised pattern recognition assumes that a training dataset is available and a classifier is designed by exploiting this a priori information. The main problem is that the structure discovered in the data can be misleading in the sense of determining the actual classes. The classification process also tries to group the samples but based on some already classified examples. In this study, LVQ-ANN is used as classifier that tries to group the samples based on the micro statistical features extracted from the samples. Figure 1 shows the pattern recognition system for glaucoma diagnosis. 
Decision

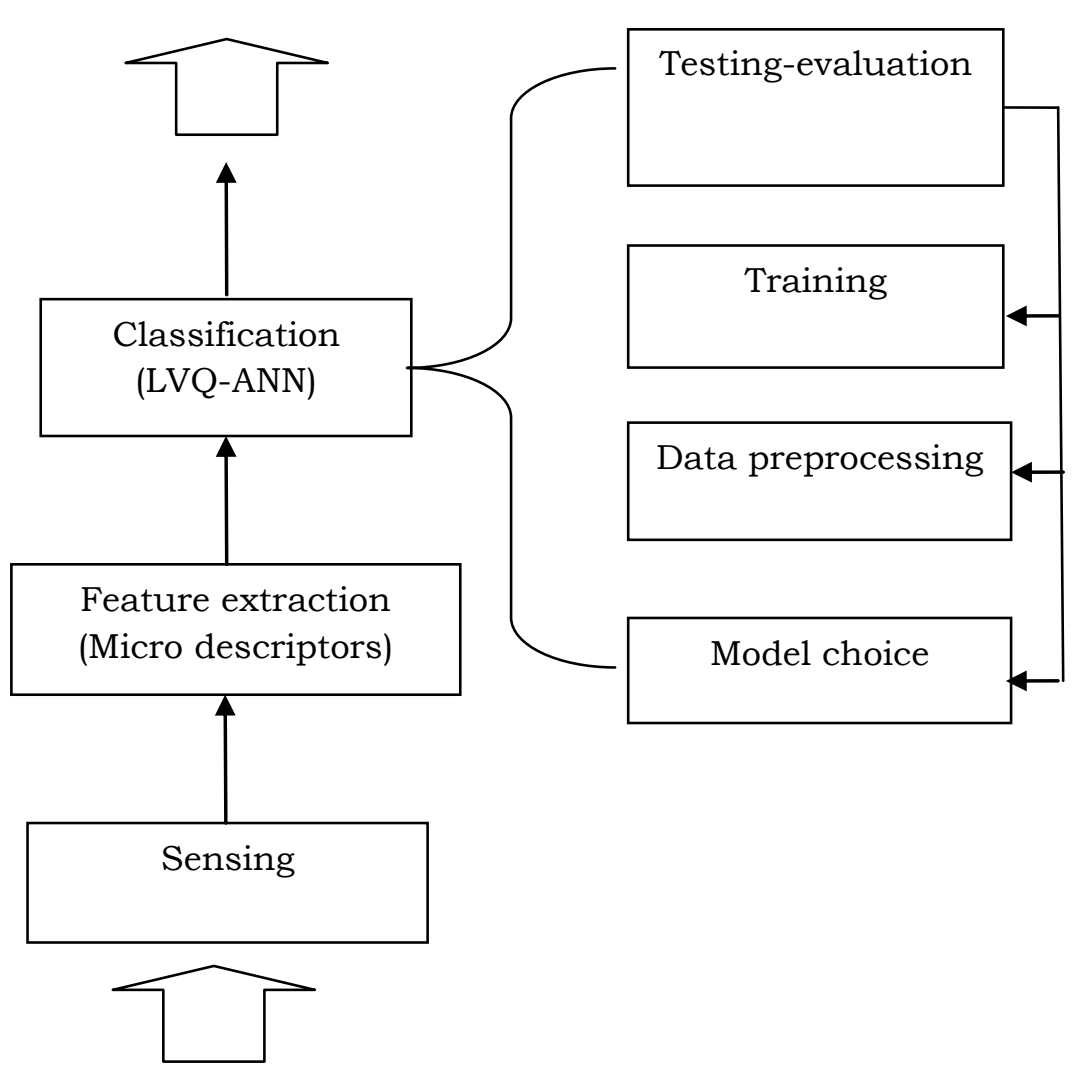

Input

Fig. 1 Pattern recognition system for glaucoma diagnosis

The proposed system for glaucoma diagnosis consists of three modules; ROI extraction, Feature extraction and Classification. The input to the systems are from three fundus image databases; DRISHTI-GS1 [13], ORIGA [14], and RIMONE [15]. The first module is applied to only DRISHTI-GS1 and ORIGA database images as RIM-ONE database provides the exact ROI (OD region only). In the second module, micro statistical descriptors are extracted using LAWS kernels and then in the final module, LVQ-ANN classifier is used to discriminate the fundus images into normal or glaucoma.

\section{A. ROI Extraction}

To increase the reliability of the LVQ-ANN based fundus image analysis, ROI extraction is employed. Whilst straightforward, the ROI extraction method makes an assumption that the brightest region is in the OD region. Based on the brightest point in the green channel, ROI is extracted. Figure 2 shows the extracted ROI from the DRISHTI-GS1 database fundus images. 

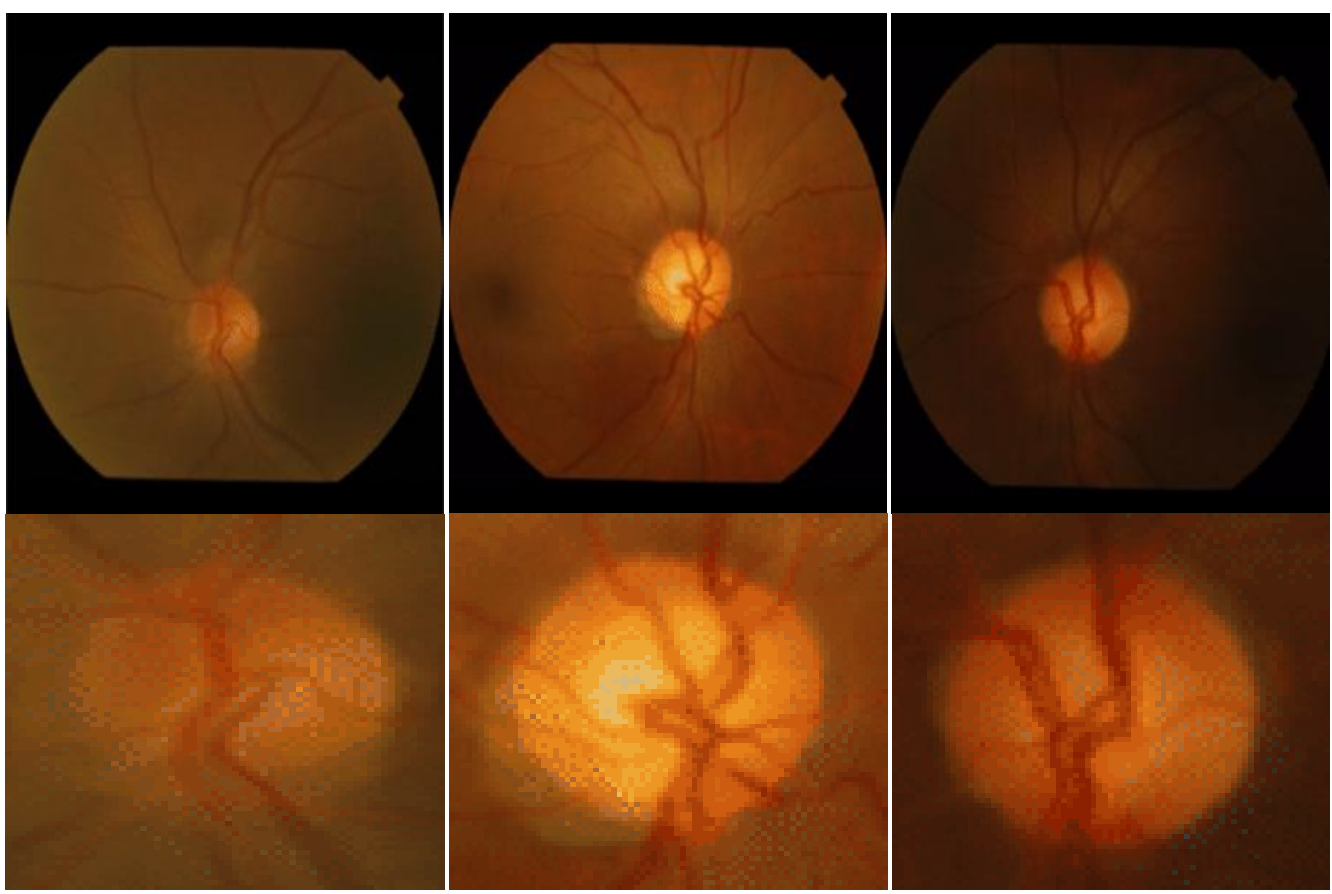

Fig. 2 ROI of DRISHTI-GS1 database fundus images

\section{B. Micro Statistical Descriptors}

LAWS [16] defined micro statistical feature descriptors which are derived from spatial filters. Features are extracted from the fundus image by convoluting it with spatial filters. The $5 \times 5$ kernels in the spatial filter are derived from the center weighted vectors. The five kernels are as follows: Level5 (L5) $=[1,4,6,4,1]$, Edge5 (E5) $=[-1,-2,0,2,1]$, Ripple5 $(R 5)=[1,-4,6,-4,1]$, Wave5 (W5) $=[-1,2,0$, $-2,1]$ and Spot5 (S5) $=[-1,0,2,0,-1]$. When multiplying these kernels, one can get 25 matrices. Then, the convolution of these matrices with the input image, 25 new images are obtained. The kernels used for the extraction of micro statistical features are shown in Figure 3. From the obtained 25 new images, texture energy measures are computed as features.

\section{LVQ-ANN Classification}

ANN is not model based but is data-driven. They are modelled along the biological neuron. These networks compute any desired function that captures the underlying structure of the data which formulates a model of the system that generated it. This function is represented by the stored weights obtained during neural network training.

The input data to a neural network can be an image(s), features or grey level values of images. The ANN uses a large training set and suitable network architecture and a training algorithm to adapt the network parameters. Some training strategies involve implementing several architectures to gain confidence in the network's ability to extract relevant information with the architecture that gives the best solution being selected. ANN represents non-linear mappings from several inputs to several output variables. The patterns in ANN are represented by 
points in a decision space which is made statistical by the variability within and between the classes.

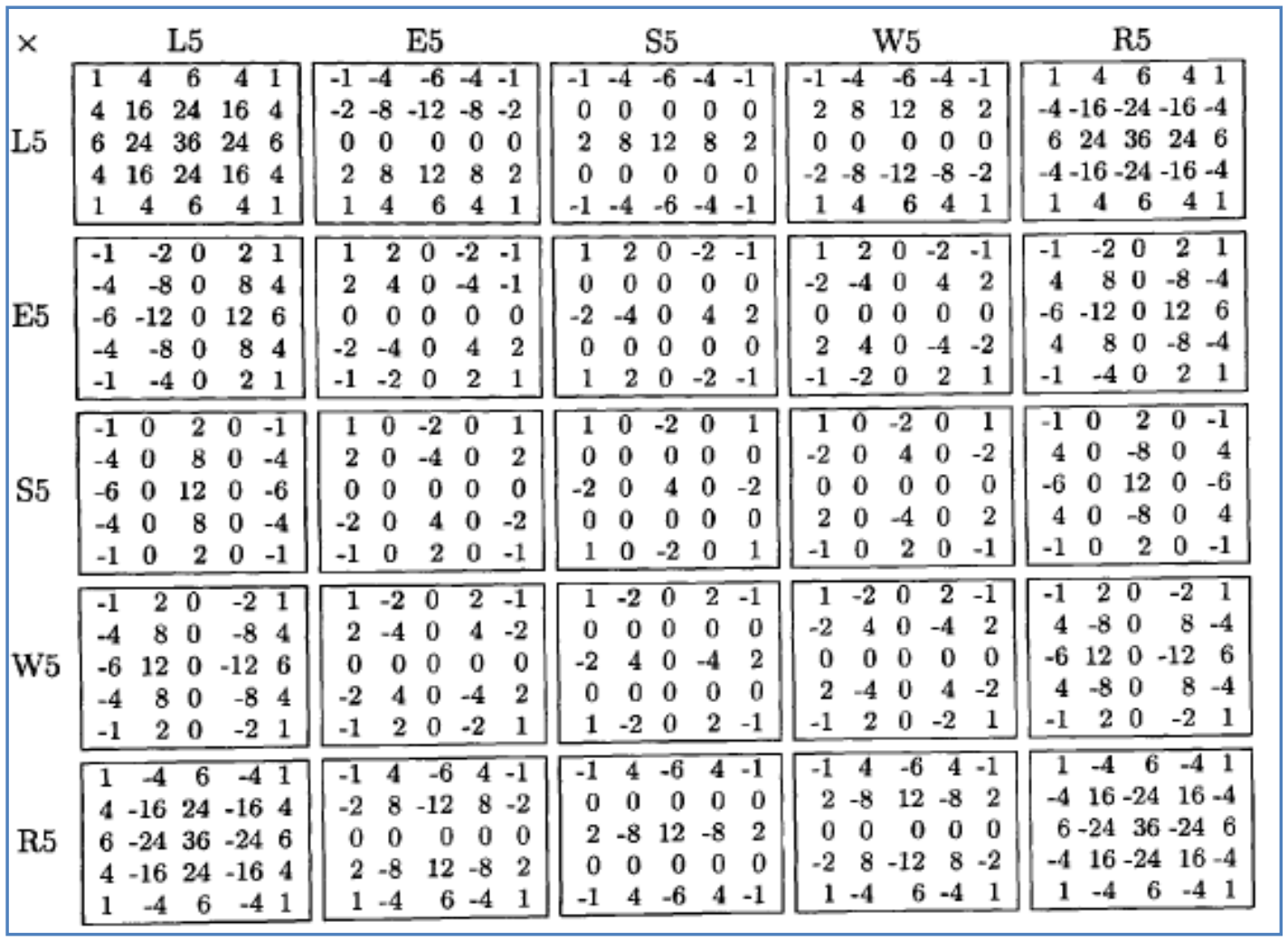

\section{Fig. 3 Kernels used for micro-statistical features}

The LVQ is a development of the Self-Organizing Map ANN architecture [17], but utilizes supervised rather than unsupervised classification. The LVQ maps the input space to the output classes by creating a number of free parameter vectors known as codebook vectors in the input space, each of which is assigned to one of the output classes. Each codebook vector is designated $m_{i}$, and any number of codebook vectors may be assigned to each output class. Each input vector $x$ is mapped to a class by comparing the current input vector with each codebook vector $m_{i}$, and assigning the input vector to the class for that codebook vector. The method of assignment is the Euclidean distance,

$$
c=\arg \min \left\{\left|x-m_{i}\right|\right\}
$$

where $x$ is the vector to be classified and is a member of the learning set of labeled samples, $H=\left\{x_{j} \mid j=1,2,3, \ldots n\right\}$. The notation $|.$.$| denotes the vector normal, and$ the notation, $\arg \min \{\ldots\}$, is the minimum value of the argument (...). By computing $c$, an input vector can be quickly and simply classified.

The values for $m_{i}$ are derived by an iterative learning process similar to the methods common to all ANN techniques whereby an initial estimate of $m_{i}$ is updated to increase the accuracy of the classification. For supervised learning, there exists a training set of vectors for which the ultimate classification is know, 


$$
H=\left\{x_{j} \mid j=1,2, \cdots, n\right\}
$$

For each element $x_{j}$ in $H, c$ is computed (Eqn. 1) for each $m_{i}$. The $m_{i}$. for $c$ is designated $m_{c}$, and this is updated for its value at iteration $k$ to:

$$
m_{c}(k+1)=m_{c}(k) \mid+\alpha(k)\left\lfloor x_{j}-m_{c}(k)\right\rfloor
$$

where $\alpha_{k}$ is an independent parameter used to control convergence of the algorithm? For stability, $0<\alpha(k)<1$, and may be constant, or iteration-dependent (generally deceasing monotonically).

If $x_{j}$ is correctly classification (i.e. $m_{c}$ for $x_{j}$ the class expected from training set $H$ ) then the next element of $H, x_{j+1}$ is computed, however, if $x_{j}$ is incorrectly classified then $m_{c}$ is updated using the equation

$$
m_{c}(k+1)=m_{c}(k) \mid-\alpha(k)\left\lfloor x_{j}-m_{c}(k)\right\rfloor
$$

By this strategy, correct classification lead to a refinement of $m_{c}$ in a direction towards $x_{j}$, whereas incorrect classification moves in the opposite direction. For those $\mathrm{m}_{\mathrm{c}}$ not close to $x_{j}$ the vector remains unchanged.

\section{RESULTS AND DISCUSSION}

This section discusses the performances of LVQ-ANN based glaucoma diagnosis using fundus images in three different databases; DRISHTI-GS1, ORIGA, and RIM-ONE. Table 1 shows the description of databases such as total number of images, number of glaucoma and normal images and their resolution in pixels. Though RIM-ONE has 38 more suspicious fundus images, the normal and glaucoma images are only considered as the proposed system is designed to discriminate normal/glaucoma images.

TABLE 1 Description of Databases

\begin{tabular}{|c|c|c|c|c|}
\hline \multirow{2}{*}{ Database } & \multirow{2}{*}{ \#total images } & \multirow{2}{*}{ Resolution } & \multicolumn{2}{|c|}{ Category } \\
\cline { 4 - 5 } & & & Glaucoma & Normal \\
\hline DRISHTI-GS1 & 101 & $2896 \times 1944$ & 70 & 31 \\
\hline ORIGA & 650 & $3072 \times 2048$ & 168 & 482 \\
\hline RIM-ONE & 169 & $2144 \times 2144$ & 39 & 92 \\
\hline
\end{tabular}

Figure 4 shows sample fundus images in the databases; ORIGA and RIMONE. Sample images in DRISHTI-GS1 database are already shown in Figure X. As the RIM-ONE database has only the OD region, the ROI extraction is not applied and features are directly extracted and fed to LVQ-ANN. 

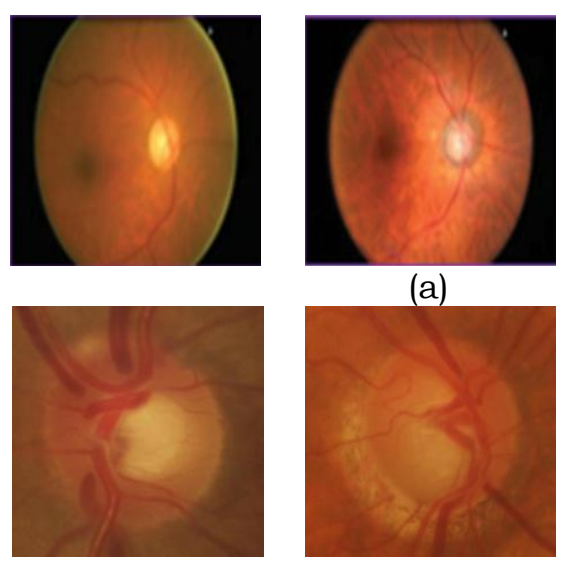

(a)

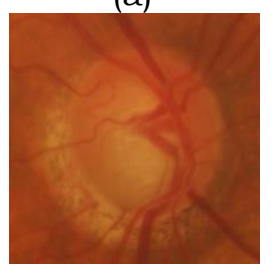

(b)
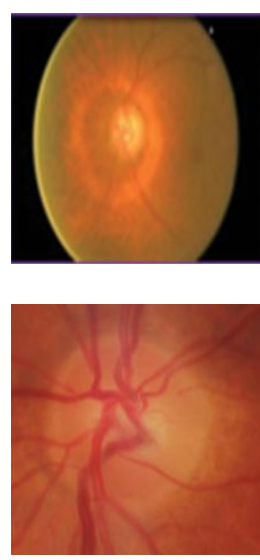

Fig. 4 Fundus images (a) ORIGA (b) RIM-ONE

The performance of LVQ-ANN system is analyzed by varying the codebook vectors. Table 2 to 4 show the performance metrics obtained by the system on DRISHTI-GS1, ORIGA and RIM-ONE databases.

TABLE 2 Performance of LVQ-ANN on DRISHTI-GS1 database

\begin{tabular}{|c|c|c|c|}
\hline Codebook vectors & Accuracy & Sensitivity & Specificity \\
\hline 5 & 77.23 & 82.86 & 64.52 \\
\hline 50 & 83.17 & 85.71 & 77.42 \\
\hline 100 & 91.09 & 92.86 & 87.10 \\
\hline 200 & $\mathbf{9 5 . 0 5}$ & $\mathbf{9 5 . 7 1}$ & $\mathbf{9 3 . 5 5}$ \\
\hline 300 & 92.08 & 92.86 & 90.32 \\
\hline
\end{tabular}

TABLE 3 Performance of LVQ-ANN on ORIGA database

\begin{tabular}{|c|c|c|c|}
\hline Codebook vectors & Accuracy & Sensitivity & Specificity \\
\hline 5 & 78.46 & 68.45 & 81.95 \\
\hline 50 & 82.62 & 77.38 & 84.44 \\
\hline 100 & 83.85 & 80.36 & 85.06 \\
\hline 200 & $\mathbf{8 5 . 3 8}$ & $\mathbf{8 3 . 3 3}$ & $\mathbf{8 6 . 1 0}$ \\
\hline 300 & 83.08 & 78.57 & 84.65 \\
\hline
\end{tabular}

TABLE 4 Performance of LVQ-ANN on RIM-ONE database

\begin{tabular}{|c|c|c|c|}
\hline Codebook vectors & Accuracy & Sensitivity & Specificity \\
\hline 5 & 79.39 & 71.79 & 82.61 \\
\hline 50 & 84.73 & 76.92 & 88.04 \\
\hline 100 & 91.60 & 89.74 & 92.39 \\
\hline 200 & $\mathbf{9 6 . 1 8}$ & $\mathbf{9 4 . 8 7}$ & $\mathbf{9 6 . 7 4}$ \\
\hline 300 & 87.79 & 82.05 & 90.22 \\
\hline
\end{tabular}


It can be seen from Table 2 to 4 that LVQ-ANN provides maximum performance when the code book size is set to 200 and least performance at 5 . For DRISHTI-GS1, the system achieves $95.05 \%$ accuracy whereas it is $85.38 \%$ and 96.18\% for ORIGA and RIM-ONE database images. Compared to three databases, the system performance on ORIGA database images is lower than DRISHTI-GS1 and RIM-ONE. This may be due to the more class imbalance in ORIGA database images. Figure 5 shows the comparison study in terms of accuracy of LVQ-ANN system on three databases.

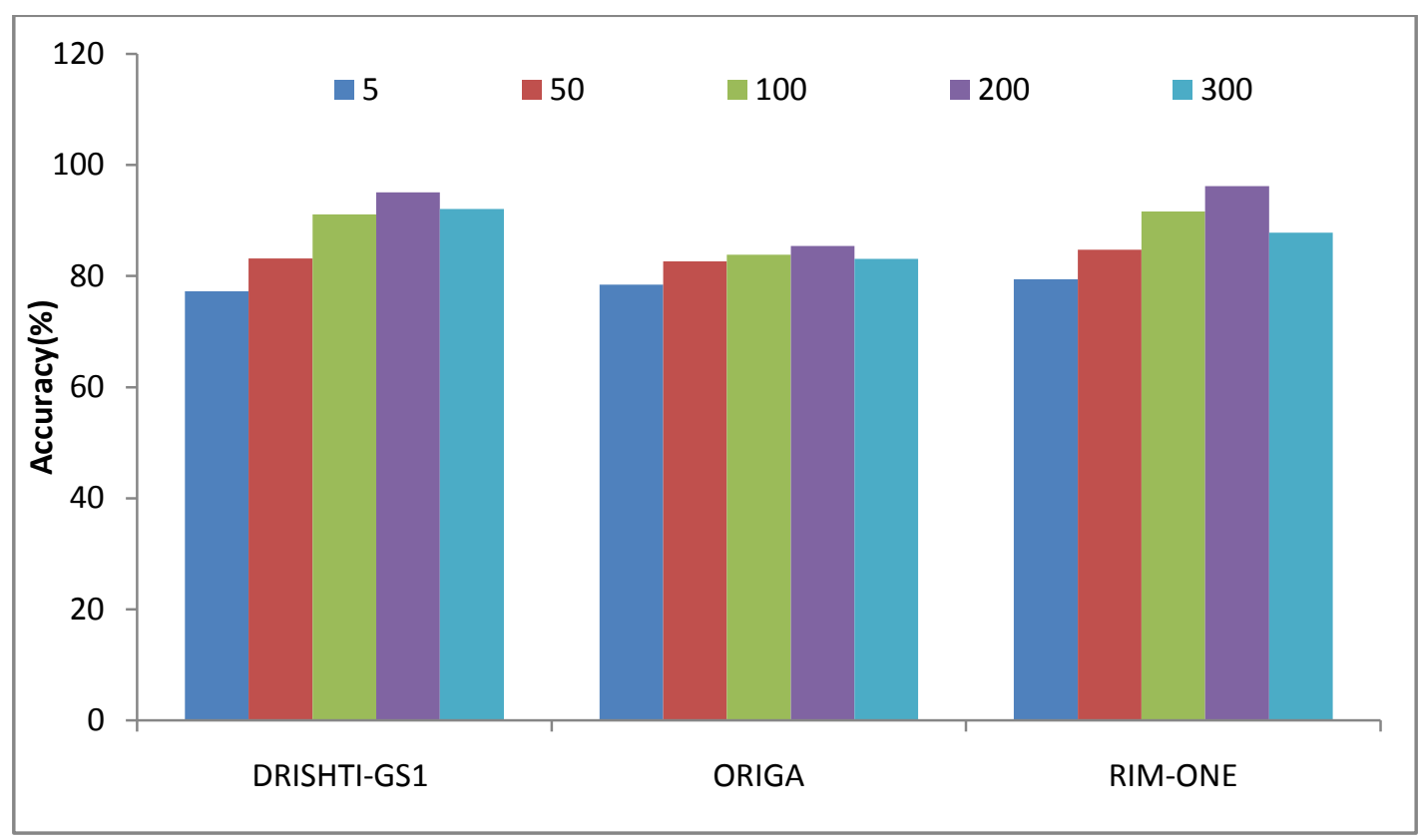

Fig. 5 Performances of LVQ-ANN system on three databases

\section{CONCLUSION}

In this study, a CAD system for the detection of glaucoma in fundus images has been developed. The proposed CAD system consists of ROI extraction, micro statistical descriptors and LVQ-ANN classification. The ROI has been extracted as a search area for the OD region where the micro statistical descriptors are extracted. Results show that LVQ-ANN classifier provides better results on three databases; DRISHTI-GS1, ORIGA, and RIM-ONE. Experimental results on these databases provide $95.05 \%, 85.38 \%$ and $96.18 \%$ accuracies respectively. The proposed CAD system is most helpful to classify fundus images for glaucoma diagnosis. As the proposed method fully automates the extraction of ROI and micro statistical descriptors, it allows the CAD system to be included in a fully automatic glaucoma diagnosis system 


\section{REFERENCES}

[1]. X. Chen, Y. Xu, D.W.K. Wong, T.Y. Wong, and J. Liu, "Glaucoma detection based on the deep convolutional neural network," 37th annual international conference of the IEEE engineering in medicine and biology society, 2015, pp. 715-718.

[2]. B. Al-Bander, W. Al-Nuaimy, M.A. Al-Taee, and Y. Zheng, "Automated glaucoma diagnosis using deep learning approach," 14th International Multi-Conference on Systems, Signals \& Devices, 2017, pp. 207-210.

[3]. M.V. Boland and H.A. Quigley, "Risk factors and open-angle glaucoma: classification and application," Journal of glaucoma, Vol. 16, No. 4, 2007, pp. 406-418.

[4]. E.A. Osman, B.A.M. Alqarni, S.S.H. AlHasani, S.S.S. Al Harbi, P.W. Gikandi, and A. Mousa, "Compliance of glaucoma patients to ocular hypotensive medications among the Saudi population," Journal of ocular pharmacology and therapeutics, Vol. 32, No. 1, 2016, pp. 50-54.

[5]. A. Ghosh, A. Sarkar, AS Ashour, D. Balas-Timar, N. Dey and V.E. Balas, "Grid color moment features in glaucoma classification," International Journal of Advanced Computer Science and Applications, Vol. 6, No. 9, 2015, pp.99-107.

[6]. A. Li, J. Cheng, D.W.K. Wong and J. Liu, "Integrating holistic and local deep features for glaucoma classification," 38th Annual International Conference of the IEEE Engineering in Medicine and Biology Society, 2016, pp. 1328-1331.

[7]. J.J. Gómez-Valverde, A. Antón, G. Fatti, B. Liefers, A. Herranz, A. Santos, and M.J. Ledesma-Carbayo, "Automatic glaucoma classification using color fundus images based on convolutional neural networks and transfer learning," Biomedical optics express, Vol. 10, No. 2, 2019, pp. 892-913.

[8]. S. Samanta, S.S. Ahmed, MAMM Salem, S.S. Nath, N. Dey, and S.S. Chowdhury, "Haralick features based automated glaucoma classification using backpropagation neural network," In Proceedings of the 3rd International Conference on Frontiers of Intelligent Computing: Theory and Applications, 2015, pp. 351-358.

[9]. A. El-Rafei, T. Engelhorn, S. Wärntges, A. Dörfler, J. Hornegger, and G. Michelson, "Glaucoma classification based on visual pathway analysis using diffusion tensor imaging," Magnetic resonance imaging, Vol. 31, No. 7, 2013, pp. 1081-1091.

[10]. F. Fink, K. Worle, P. Gruber, A.M. Tome, J.M. Gorriz-Saez, C.G. Puntonet, and E.W. Lang, "ICA analysis of retina images for glaucoma classification," 30th Annual International Conference of the IEEE Engineering in Medicine and Biology Society, 2008, pp. 4664-4667.

[11]. D. Yadav, M.P. Sarathi and M.K. Dutta, "Classification of glaucoma based on texture feature using neural networks," Seventh International Conference on Contemporary Computing, 2014, pp. 109-112.

[12]. L. Li, M. Xu, H. Liu, Y. Li, X. Wang, L. Jiang, and N. Wang, "A large-scale database and a CNN model for attention-based glaucoma detection," IEEE transactions on medical imaging, Vol. 39, No. 2, 2019, pp.413-424.

[13]. J. Sivaswamy, S.R. Krishnadas, G.D. Joshi, M. Jain, and AUS. Tabish, "Drishti-gs: Retinal image dataset for optic nerve head (onh) segmentation," IEEE $11^{\text {th }}$ International Symposium on Biomedical Imaging, 2014, pp. 5356.

[14]. Z. Zhang, F.S. Yin, J. Liu, W.K. Wong, N.M. Tan, B.H. Lee, J. Cheng, and T.Y. Wong, "Origa-light: An online retinal fundus image database for 
glaucoma analysis and research," Annual International Conference of the IEEE Engineering in Medicine and Biology, 2010, pp. 3065-3068.

[15]. F. Fumero, S. Alayón, J.L. Sanchez, J. Sigut, and M. Gonzalez-Hernandez, "RIM-ONE: An open retinal image database for optic nerve evaluation," $24^{\text {th }}$ International Symposium on Computer-Based Medical Systems, 2011; pp. $1-6$.

[16]. K.I Laws, "Rapid texture identification," International Society for Optics and Photonics, Vol. 238, 1980, pp. 376-381.

[17]. T. Kohonen, J. Kangas, J. Laaksonen, and K. Torkkola, "LVQ PAK: A program package for the correct application of Learning Vector Quantization algorithms," International Joint Conference on Neural Networks, Vol. 92, 1992, pp. 725-730. 